\title{
Long-Term Antipsychotic Polypharmacy in the VA Health System: Patient Characteristics and Treatment Patterns
}

\author{
Julie A. Kreyenbuhl, Pharm.D., Ph.D., Marcia Valenstein, M.D., M.S., John F. McCarthy, \\ Ph.D., M.P.H., Dara Ganoczy, M.P.H., and Frederic C. Blow, Ph.D. \\ Dr. Kreyenbuhl is affiliated with the Division of Services Research, Department of Psychiatry, \\ University of Maryland School of Medicine, 737 W. Lombard St., Baltimore, MD, 21201 \\ (jkreyenb@psych.umaryland.edu). She is also with the Department of Veterans Affairs (VA) \\ Capitol Healthcare Network (VISN 5) Mental Illness Research, Education, and Clinical Center, \\ Baltimore, Maryland. Dr. Valenstein, Dr. McCarthy, Ms. Ganoczy, and Dr. Blow are with the VA \\ Serious Mental Illness Treatment Research and Evaluation Center, Ann Arbor, Michigan. Dr. \\ Valenstein, Dr. McCarthy, and Dr. Blow are also with the Department of Psychiatry, University of \\ Michigan Medical School, Ann Arbor. An earlier version of this article was presented at the \\ International Congress on Schizophrenia Research Bi-Annual Meeting, Savannah, Georgia, April \\ 2-6, 2005.
}

\section{Abstract}

Objective-Although antipsychotic polypharmacy is being prescribed with increasing frequency, few studies have described patient characteristics and treatment patterns associated with long-term use of this treatment strategy.

Methods-By using data from the National Psychosis Registry of the Department of Veterans Affairs, 5,826 patients with schizophrenia or schizoaffective disorder who received long-term antipsychotic polypharmacy (simultaneous treatment with two or more antipsychotics for 90 or more days) during fiscal year 2000 and 39,745 patients who received long-term antipsychotic monotherapy were identified. By using multivariate regression models, patient demographic and clinical characteristics, antipsychotic dosages, and use of antiparkinson and adjunctive psychotropic medications were compared between the two groups.

Results-Patients were more likely to receive antipsychotic polypharmacy if they were younger, were unmarried, had a military service-connected disability, had schizophrenia rather than schizoaffective disorder, or had greater use of inpatient and outpatient mental health services. Patients were less likely to receive antipsychotic polypharmacy if they were African American, had concurrent diagnoses of depression or substance use disorder, or had greater medical comorbidity. For most antipsychotics, dosages prescribed for patients receiving polypharmacy were the same or modestly higher than those prescribed for patients receiving monotherapy. Patients given prescriptions for polypharmacy were more likely to receive antiparkinson medications, antianxiety agents, and mood stabilizers and equally likely to receive concurrent treatment with antidepressants.

Conclusions-Long-term antipsychotic polypharmacy appears to be reserved for more severely ill patients with psychotic symptoms rather than mood symptoms. These patients may experience increased adverse effects as a result of excess antipsychotic exposure.

Many patients with schizophrenia have symptoms that either do not or only partly respond to monotherapy with antipsychotic medications, including clozapine (1), the only

The authors report no competing interests. 
antipsychotic medication with demonstrated efficacy for treatment-resistant schizophrenia (2). The sustained use of combinations of antipsychotic medications- "antipsychotic polypharmacy"-is an increasingly common treatment strategy (3-14). However, evaluations of the efficacy of antipsychotic combinations have been limited to small randomized controlled trials of clozapine augmentation (15-19) and several case reports and uncontrolled investigations of other combinations of antipsychotic agents (20-21).

Although not widely studied, treatment with multiple antipsychotic agents may present risks to patients, such as increased adverse effects and drug interactions, decreased treatment adherence, increased costs for patients and health care systems, and a possible increased risk of mortality $(22,23)$. Given these concerns, long-term use of antipsychotic combinations is recommended only as a last-line treatment strategy (24).

One rationale for treatment with antipsychotic combinations is that lower dosages of agents having different side effects profiles may achieve adequate efficacy with fewer side effects than when a single agent is used in higher dosages (20). However, several studies indicate that patients given prescriptions for antipsychotic combinations receive similar or higher total antipsychotic dosages than patients given prescriptions for monotherapy $(3,6,10)$. Only two studies have compared the dosage patterns of individual antipsychotic agents when prescribed in combination and in monotherapy $(7,14)$. In these single-site studies the dosages of second-generation agents used in combination versus the dosages of such agents used as a monotherapy were reported to be similar. Dosages of first-generation antipsychotic agents, commonly prescribed as a part of combination regimens, have not been reported.

When two or more antipsychotics are used in combination in standard dosages, excessive antipsychotic exposure may lead to significant short-and long-term adverse effects. However, only a few reports have evaluated whether patients given prescriptions for antipsychotic combinations experience adverse reactions, such as extrapyramidal side effects (4), or receive antiparkinson medications more frequently, compared with those given prescriptions for monotherapy $(7,14)$. In addition, the extent to which patients given prescriptions for long-term antipsychotic polypharmacy receive additional psychotropic medications has not been carefully evaluated. Given that simultaneous within- and betweenmedication class polypharmacy may increase risks of adverse effects and drug interactions and can also substantially increase costs, more information is needed regarding concurrent prescription patterns.

Comparisons across prior studies of the patient factors and treatment patterns associated with antipsychotic polypharmacy are complicated by inconsistencies in study design, diversity in the patient populations studied, and operational definitions of polypharmacy (25). The most commonly used methods for identifying patients given prescriptions for polypharmacy often fail to differentiate between appropriate prescriptions of multiple agents on a short-term temporary basis and long-term combination antipsychotic treatment regimens, which may increase risks to patients. In this retrospective study we evaluated Department of Veterans Affairs (VA) patients with schizophrenia or schizoaffective disorder who were given prescriptions for sustained treatment with antipsychotic combinations (90 or more days). In order to identify the patient demographic and clinical factors associated with long-term treatment, we compared these patients to those given prescriptions for sustained antipsychotic monotherapy. We also compared antipsychotic dosage patterns, use of antiparkinson medications, and use of adjunctive psychotropic agents prescribed between the two patient groups. 


\section{Methods}

\section{Data sources}

This study used data from 1999 to 2001 from the VA National Psychosis Registry (NPR) (26-28), a longitudinal resource developed and maintained by the national VA Serious Mental Illness Treatment Research and Evaluation Center in Ann Arbor, Michigan. The NPR contains administrative encounter records of all in-patient and outpatient health services provided to veterans with psychotic disorders in VA facilities. Records also include information on patient demographic characteristics, ICD- 9 diagnostic codes, and medication use. Pharmacy data incorporated into the NPR were obtained from the VA Pharmacy Benefits Management Strategic Health Group and include all outpatient prescriptions (excluding long-acting injectable antipsychotic medications) dispensed from VA facilities. The study was reviewed by the University of Maryland Institutional Review Board and qualified as exempt research.

\section{Study samples}

By using the diagnostic codes from inpatient and outpatient encounter data, we identified 77,243 patients with a diagnosis of schizophrenia or schizoaffective disorder during both fiscal year (FY) 1999 and FY 2000. Patients with schizophrenia had ICD-9 codes of 295.0, 295.1, 295.2, 295.3, 295.4, 295.6, 295.8, or 295.9 recorded during FY 2000 in a majority of their encounters (relative to other $I C D-9$ codes indicative of other serious mental illness), whereas patients with schizoaffective disorder had an ICD-9 code of 295.7 occurring most frequently (26-28). We excluded 1,798 patients for whom there were fewer than 90 observation days during FY 2000, as a result of such factors as death or institutionalization, and 14,188 patients who did not have at least one prescription for an oral antipsychotic medication during FY 2000.

From the remaining 61,257 patients, we identified 5,826 (9.5\%) who received long-term antipsychotic polypharmacy, defined as receipt of two or more oral antipsychotic medications simultaneously for at least 90 consecutive days during the study period (25). Patients who did not meet this criterion but who had at least 90 consecutive days of treatment with the same oral antipsychotic medication during the study period comprised the monotherapy comparison group $(\mathrm{N}=39,745)$.

\section{Measures}

Predictors of long-term antipsychotic polypharmacy-We first examined associations between the receipt of long-term antipsychotic polypharmacy and patient demographic and clinical characteristics. We selected variables for inclusion in the statistical model on the basis of the results of previous studies of antipsychotic polypharmacy in addition to other factors likely related to antipsychotic treatment choice.

We included in the model patient age $(8,12,13)$, gender (5), race (white, African American, Hispanic, other [for example, Asian, Native American, or Pacific Islander], or unknown race) $(8,11,13)$, marital status (married versus not married) (11), and an indicator of whether the patient had a military service-connected disability (13). By using $I C D-9$ diagnostic codes, we included clinical indicators for whether the patient was diagnosed as having schizophrenia (versus schizoaffective disorder), major depression (13), a substance use disorder $(5,13)$, or posttraumatic stress disorder (PTSD). We also included an indicator of whether the patient had a psychiatric hospitalization in $1999(8,13)$ and the number of outpatient mental health visits during the study period (5). Finally, we included an indicator of medical comorbidity (5), by using the Charlson Index (29). The Charlson Index score is 
the sum of weighted values assigned to 19 categories of comorbidity. Because the median score was zero, we categorized scores as 0 or $\geq 1$.

Antipsychotic dosing-We calculated average antipsychotic dosages for each patient by examining the first 90 days of antipsychotic polytherapy or monotherapy during the study period. We converted dosages of all first-generation antipsychotic medications to chlorpromazine equivalents, the approximate dosage equivalent to a standard dosage of 100 $\mathrm{mg}$ of chlorpromazine, on the basis of a widely used conversion table (30). We used standard milligram dosages for all second-generation antipsychotic medications prescribed during the study period.

\section{Use of antiparkinson agents and adjunctive psychotropic medications-We}

constructed dichotomous indicators of receipt of antiparkinson agents, antidepressant agents, antianxiety agents, or mood-stabilizing agents during the first 90 days of each patient's antipsychotic treatment during the study period.

\section{Analyses}

We used a hierarchical data analytic approach because of the clustered nature of our data, with patient observations nested within VA facilities. We first used generalized linear mixed models to examine multivariate relationships between the dependent variable (long-term antipsychotic polypharmacy) and patient demographic characteristics (age, gender, race, marital status, and military service-connected disability status) and clinical characteristics (schizophrenia versus schizoaffective disorder, depression, substance use disorder, PTSD, psychiatric hospitalization in the past year, total outpatient mental health visits during the study period, and score on the Charlson Index).

The distribution of antipsychotic dosage was highly skewed. We therefore used the Wilcoxon rank-sum test to determine whether dosages for each antipsychotic medication differed for patients who did or did not receive long-term combination antipsychotic treatment. Next, by using hierarchical multivariate regression models similar to those described above, we examined the relationship between the dependent variable (antipsychotic dosage) and a dichotomous indicator of whether the patient was given prescriptions for long-term antipsychotic polypharmacy, adjusting for patient demographic and clinical characteristics. Having converted dosages for each first-generation antipsychotic medication to standardized chlorpromazine equivalents, we then evaluated antipsychotic dosage using a single regression model for first-generation agents. For each of the secondgeneration antipsychotic medications, we constructed separate models.

Finally, we constructed hierarchical multivariate logistic regression models to evaluate whether treatment with long-term antipsychotic poly-pharmacy was associated with an increased likelihood of receiving antiparkinson medications and each class of adjunctive psychotropic medications. We evaluated separate models for each drug class, and the models were adjusted for the patient demographic and clinical characteristics described above. All study analyses were completed by using SAS, version 9.1 .

\section{Results}

In Table 1 we present the results of the multivariate analyses examining the associations between patient factors and the likelihood of receiving long-term antipsychotic polypharmacy. Patients were more likely to have received long-term antipsychotic polypharmacy if they were younger, were unmarried, had a military service-connected disability, or were diagnosed as having schizophrenia rather than schizoaffective disorder. Patients were also more likely to receive antipsychotic polypharmacy if they had a 
psychiatric hospitalization in the prior year or had more outpatient mental health visits. Patients who were African American were less likely than white patients to have been given pre scriptions for polypharmacy. Co-occurring depression or a substance use disorder was associated with decreased odds of receiving antipsychotic polypharmacy, whereas comorbid PTSD was not significantly associated with the likelihood of receiving combination antipsychotic treatment. Patients with a higher burden of medical comorbidity, as measured by the Charlson Index, had reduced odds of receiving long-term antipsychotic polypharmacy.

In bivariate analyses of second-generation antipsychotics, dosages of olanzapine, quetiapine, and risperi-done were higher when used concurrently with other antipsychotic agents, compared with when they were prescribed alone, although the absolute differences in dosage levels were small (Table 2). Dosages for clozapine did not differ significantly between the two groups in bivariate analyses. The results of the multivariate analyses for each agent were consistent with the results of the bivariate analyses.

Among the first-generation antipsychotic medications, dosages of chlorpromazine were lower and dosages of thiothixene and haloperidol were higher among patients given prescriptions for antipsychotic combinations in bivariate analyses, compared with patients receiving monotherapy. Dosages of all other first-generation agents did not differ significantly between the groups. However, when all first-generation antipsychotics were considered, multivariate analyses indicated that patients given prescriptions for polypharmacy received lower dosages of first-generation agents, compared with patients given prescriptions for monotherapy (adjusted odds ratio [AOR] $=.93$, 95\% confidence interval $[\mathrm{CI}]=.90-.96, \mathrm{p}<.001)$. This may be explained by the confounding effects of age. Older patients were less likely to be given prescriptions for long-term polypharmacy. The modal dosage of first-generation agents among patients younger than 64 years was 500 chlorpromazine equivalents both for those given prescriptions for polypharmacy and for those given prescriptions for monotherapy; among patients 65 years and older, however, the modal dosage for both groups was 100 chlorpromazine equivalents. After the analysis adjusted for differences by age and other model covariates, patients given prescriptions for polypharmacy received somewhat lower dosages of first-generation agents (as a group), compared with monotherapy patients.

Figure 1 presents the proportions of patients given prescriptions for antiparkinson medications, antidepressants, antianxiety agents, and mood stabilizer medications, by receipt of long-term antipsychotic polypharmacy. Although $36 \%(\mathrm{~N}=14,384)$ of patients given prescriptions for mono-therapy received concurrent antiparkinson medications, these agents were received by $50 \%(\mathrm{~N}=2,894)$ of patients given prescriptions for an tipsychotic polypharmacy. In multivariate analyses that adjusted for patient demographic and clinical characteristics, patients given prescriptions for antipsychotic polypharmacy were more likely to receive concurrent treatment with antiparkinson agents than patients given prescriptions for monotherapy ( $\mathrm{AOR}=1.67, \mathrm{CI}=1.58-1.77, \mathrm{p}<.001)$. The results of multivariate analyses indicated that the odds of receiving antidepressant medications did not differ by antipsychotic polypharmacy status. In contrast, patients receiving long-term polypharmacy had a higher likelihood of prescription of antianxiety medications, compared with patients not given prescriptions for polypharmacy (2,409 patients receiving polypharmacy, or $41 \%$, compared with 12,992 patients receiving monotherapy, or $33 \%$; $\mathrm{AOR}=1.52,95 \% \mathrm{CI}=1.43-1.61, \mathrm{p}<.001)$.

Concurrent treatment with a mood stabilizer was observed among $37 \%(\mathrm{~N}=2,148)$ of patients given prescriptions for antipsychotic polypharmacy, compared with $27 \%$ $(\mathrm{N}=10,797)$ of patients given prescriptions for monotherapy. Patients given prescriptions for 
antipsychotic polypharmacy were approximately one-and-one-half times more likely than those given prescriptions for monotherapy to receive these additional treatments $(\mathrm{AOR}=1.45,95 \% \mathrm{CI}=1.36-1.55, \mathrm{p}<.001)$.

\section{Discussion}

This study represents one of the first investigations of the characteristics of patients with schizophrenia who received antipsychotic polypharmacy on a long-term basis. Further, it provides information not previously reported on the attributes of these medication regimens. We observed that sustained coprescription of antipsychotic medications-a practice for which there is little evidence regarding safety or efficacy—appears to be generally reserved for patients who are most severely ill. Consistent with earlier reports $(8,11-13)$, our study found that patients were more likely to receive antipsychotic polypharmacy if they were younger, unmarried, or had greater use of inpatient and outpatient mental health services in the prior year.

In addition, our data suggest that treatment with antipsychotic combinations is targeted to residual psychotic symptoms rather than mood-related symptoms, as evidenced by our findings that patients with schizoaffective disorder or a concurrent diagnosis of depression were less likely to be given prescriptions for antipsychotic combinations. In addition, patients given prescriptions for antipsychotic polypharmacy were equally likely as other patients to receive antidepressant medications, yet they were more likely to be given prescriptions for antianxiety or mood stabilizer medications, which are sometimes used to treat residual psychotic symptoms. These findings are consistent with a recent study demonstrating that patients receiving antipsychotic combinations exhibited more positive and excited symptoms than patients given prescriptions for monotherapy (3) and also support clinicians' reports of using polypharmacy for refractory psychotic symptoms $(9,31)$.

Although our findings imply that the most severely ill patients were given prescriptions for antipsychotic combinations, we are unable to comment on the appropriateness of these treatment choices and their outcomes, given the current study's cross-sectional design and lack of access to detailed psychopharmacologic treatment histories (for example, prior use of clozapine) and outcomes (for example, subsequent psychiatric hospitalization or use of emergency department services). Both the appropriateness and outcomes of antipsychotic polypharmacy are important questions that merit further investigation, possibly by using electronic health records from the VA or other systems of care in a longitudinal design.

Regarding what is currently known about the efficacy of antipsychotic combinations, the few randomized, double-blind studies conducted, all of which tested augmentation of clozapine, have reported largely negative results (15-19). Further, two case-control studies $(3,4)$ of inpatients found that treatment with various antipsychotic combinations confers no benefit with respect to symptoms, functioning, or length of hospital stay, relative to treatment with monotherapy. As such, combining antipsychotics, even among chronically ill patients who may have failed other evidence-based treatments, is difficult to justify given the current state of the evidence.

In addition, the results of this study add to the concerns that have been raised regarding the safety of antipsychotic polypharmacy. We did not find support for the hypothesis that patients given prescriptions for polypharmacy receive lower dosages of individual agents in order to maintain efficacy while minimizing side effects. When used in combination, the second-generation antipsychotic agents were prescribed in similar or modestly higher dosages than the dosages considered standard for monotherapy in clinical practice. Among the individual first-generation agents, only chlorpromazine was prescribed in lower dosages 
for patients who received polypharmacy, whereas two commonly prescribed first-generation agents (haloperidol and thiothixene) were prescribed in higher dosages to these patients.

Although as a group, first-generation agents were prescribed in modestly lower dosages among patients receiving polypharmacy, compared with those receiving monotherapy, the median and modal dosages prescribed to the two groups were very similar. Only the subgroup of older patients, accounting for less than a quarter of the sample, was given pre scriptions for relatively low dosages of first-generation agents. Because most of the antipsychotic combinations prescribed in this study consisted of at least one (and sometimes multiple) first-generation antipsychotic agents (25), serious concerns are raised about the potential for adverse effects resulting from excessive antipsychotic exposure occurring over extended periods, which may begin early in a patient's treatment.

Consistent with this finding and the results of previous reports $(7,14)$, patients given prescriptions for long-term antipsychotic polypharmacy were more likely to receive antiparkinson medications than patients given prescriptions for monotherapy. These results suggest that patients may experience increased frequency of troubling extrapyramidal side effects while on polypharmacy regimens, necessitating intervention. These findings, in turn, raise concerns about other polypharmacy-related adverse effects that may arise from excessive dopamine D2 blockade, including akathisia, tardive dyskinesia, and prolactin elevation $(17,20)$

On the other hand, our data also suggest that in some respects, clinicians may be aware of certain potential risks associated with prescription of multiple antipsychotic medications. Patients who were older and had a higher burden of medical comorbidity were less likely to be given prescriptions for sustained treatment with antipsychotic combinations. Although it may be that patients with these characteristics have less severe psychotic symptoms, this may also reflect clinician recognition that co-occurring medical disorders may be worsened by the side effects of multiple antipsychotic agents and treatment may be complicated by interactions among multiple agents. Patients with diagnoses of substance use disorder were also less likely to receive antipsychotic combinations, potentially indicating clinicians' concerns about the ability of such patients to adhere to complicated medical regimens.

Major strengths of this study include the large study sample drawn from an ethnically and geographically diverse treated population with schizophrenia. However, some aspects of the study design, such as the predominately male population studied within a single system of care, may have limited the generalizability of our findings. Although we are unaware of any systemwide prescribing guidelines in the VA that may have influenced treatment patterns, our findings require replication in other treatment settings. More work is also needed to address the appropriateness and effectiveness of antipsychotic combinations.

\section{Conclusions}

The results of this observational analysis suggest that patients given prescriptions for antipsychotic polypharmacy on a long-term basis are more severely ill with psychotic symptoms rather than mood symptoms. These patients likely require enhanced monitoring for adverse effects that may result from excess antipsychotic exposure.

\section{Acknowledgments}

This work was supported by a National Institute of Mental Health Research Career Award (K01-MH066009) to Dr. Kreyenbuhl, a Department of Veterans Affairs Health Services Research and Development Service (HSR\&D) Advanced Research Career Development Award (98-350-01) to Dr. Valenstein, and a Department of Veterans Affairs HSR\&D Merit Review Entry Program Award (MRP 03-320) to Dr. McCarthy. 


\section{References}

1. Canales PL, Olsen J, Miller AL, et al. Role of antipsychotic polypharmacotherapy in the treatment of schizophrenia. CNS Drugs. 1999; 12:179-188.

2. Kane J, Honigfeld G, Singer J, et al. Clozapine for the treatment-resistant schizophrenic: a doubleblind comparison with chlorpromazine. Archives of General Psychiatry. 1988; 45:789-796.

[PubMed: 3046553]

3. Centorrino F, Fogarty KV, Sani G, et al. Use of combinations of antipsychotics: McLean Hospital inpatients, 2002. Human Psychopharmacology: Clinical and Experimental. 2005; 20:485-492. [PubMed: 16116665]

4. Centorrino F, Goren JL, Hennen J, et al. Multiple versus single antipsychotic agents for hospitalized patients: case control study of risks versus benefits. American Journal of Psychiatry. 2004; 161:700-706. [PubMed: 15056517]

5. Ganguly R, Kotzan JA, Miller S, et al. Prevalence, trends, and factors associated with antipsychotic polypharmacy among Medicaid-eligible schizophrenia patients, 1998-2000. Journal of Clinical Psychiatry. 2004; 65:1377-1388. [PubMed: 15491242]

6. Humberstone V, Wheeler A, Lambert T. An audit of outpatient antipsychotic usage in the three health sectors of Auckland, New Zealand. Australian and New Zealand Journal of Psychiatry. 2004; 38:240-245. [PubMed: 15038803]

7. Procyshyn RM, Thompson B. Patterns of antipsychotic utilization in a tertiary care psychiatric institution. Pharmacopsychiatry. 2004; 37:12-17. [PubMed: 14750043]

8. Jaffe AB, Levine J. Antipsychotic medication coprescribing in a large state hospital system. Pharmacoepidemiology and Drug Safety. 2003; 12:41-48. [PubMed: 12616846]

9. Tapp A, Wood AE, Secrest L, et al. Combination antipsychotic therapy in clinical practice. Psychiatric Services. 2003; 54:55-59. [PubMed: 12509667]

10. Tempier RP, Pawliuk NH. Conventional, atypical, and combination antipsychotic prescriptions: a 2-year comparison. Journal of Clinical Psychiatry. 2003; 64:673-679. [PubMed: 12823082]

11. Covell NH, Jackson CT, Evans AC, et al. Antipsychotic prescribing practices in Connecticut's public mental health system: rates of changing medications and prescribing styles. Schizophrenia Bulletin. 2002; 28:17-29. [PubMed: 12047017]

12. Weissman EM. Antipsychotic prescribing practices in the Veterans Healthcare AdministrationNew York Metropolitan Region. Schizophrenia Bulletin. 2002; 28:31-42. [PubMed: 12047020]

13. Leslie DL, Rosenheck RA. Use of pharmacy data to assess quality of pharmacotherapy for schizophrenia in a national health care system: individual and facility predictors. Medical Care. 2001; 39:923-933. [PubMed: 11502950]

14. Procyshyn RM, Kennedy NB, Tse G, et al. Antipsychotic polypharmacy: a survey of discharge prescriptions from a tertiary care psychiatric institution. Canadian Journal of Psychiatry. 2001; 46:334-339.

15. Honer WG, Thornton AE, Chen EYH, et al. Clozapine alone versus clozapine and risperidone with refractory schizophrenia. New England Journal of Medicine. 2006; 354:472-482. [PubMed: 16452559]

16. Josiassen RC, Joseph A, Kohegyi E, et al. Clozapine augmented with risperidone in the treatment of schizophrenia: a randomized, double-blind, placebo-controlled trial. American Journal of Psychiatry. 2005; 162:130-136. [PubMed: 15625211]

17. Anil Yagcioglu AE, Kivircik Akdede BB, Turgut TI, et al. A double-blind controlled study of adjunctive treatment with risperidone in schizophrenic patients partially responsive to clozapine: efficacy and safety. Journal of Clinical Psychiatry. 2005; 66:63-72. [PubMed: 15669890]

18. Shiloh R, Zemishlany A, Aizenberg D, et al. Sulpiride augmentation in people with schizophrenia partially responsive to clozapine. British Journal of Psychiatry. 1997; 171:569-573. [PubMed: 9519099]

19. Potter WZ, Ko GN, Zhang LD, et al. Clozapine in China: a review and preview of US/PRC collaboration. Psychopharmacology (Berlin). 1989; 99(suppl):S87-S91. [PubMed: 2682733] 
20. Freudenreich O, Goff DC. Antipsychotic combination therapy in schizophrenia: a review of efficacy and risks of current combinations. Acta Psychiatrica Scandinavica. 2002; 106:323-330. [PubMed: 12366465]

21. Stahl SM, Grady MM. A critical review of atypical antipsychotic utilization: comparing monotherapy with polypharmacy and augmentation. Current Medicinal Chemistry. 2004; 11:313327. [PubMed: 14965234]

22. Joukamaa M, Heliovaara M, Knekt P, et al. Schizophrenia, neuroleptic medication and mortality. British Journal of Psychiatry. 2006; 188:122-127. [PubMed: 16449697]

23. Waddington JL, Youssef JA, Kinsella A. Mortality in schizophrenia: Antipsychotic polypharmacy and absence of adjunctive anticholinergics over the course of a 10-year prospective study. British Journal of Psychiatry. 1998; 173:325-329. [PubMed: 9926037]

24. Miller AL, Hall CS, Buchanan RW, et al. The Texas Medication Algorithm Project antipsychotic algorithm for schizophrenia: 2003 update. Journal of Clinical Psychiatry. 2004; 65:500-508. [PubMed: 15119912]

25. Kreyenbuhl J, Valenstein M, McCarthy JF, et al. Long-term combination antipsychotic treatment in VA patients with schizophrenia. Schizophrenia Research. 2006; 84:90-99. [PubMed: 16631354]

26. Blow, FC.; McCarthy, JF.; Valenstein, M., et al. Care in the VHA for Veterans With Psychosis 1999. Veterans Health Administration Health Services Research and Development; Ann Arbor, Mich: 2000.

27. Blow, FC.; McCarthy, JF.; Valenstein, M., et al. Care in the VHA for Veterans With Psychoses FY00: 2nd Annual National Psychoses Registry Report. Veterans Health Administration Health Services Research and Development; Ann Arbor, Mich: 2001.

28. Blow, FC.; McCarthy, JF.; Valenstein, M., et al. Care in the VHA for Veterans With Psychoses FY01: 3rd Annual National Psychoses Registry Report. Veterans Health Administration Health Services Research and Development; Ann Arbor, Mich: 2001.

29. Charlson ME, Pompei P, Ales KL, et al. A new method of classifying prognostic comorbidity in longitudinal studies: development and validation. Journal of Chronic Disease. 1987; 40:373-383.

30. Lehman AF, Kreyenbuhl J, Buchanan RW, et al. The Schizophrenia Patient Outcomes Research Team (PORT): updated treatment recommendations 2003. Schizophrenia Bulletin. 2004; 30:193217. [PubMed: 15279040]

31. Sernyak MJ, Rosenheck R. Clinicians' reasons for antipsychotic coprescribing. Journal of Clinical Psychiatry. 2004; 65:1597-1600. [PubMed: 15641863] 


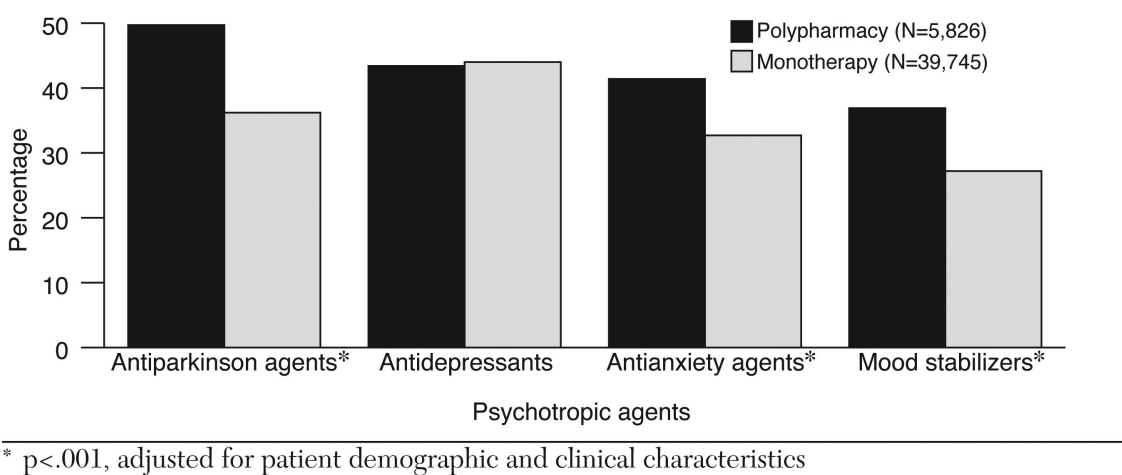

Figure 1.

Use of antiparkinson and adjunctive psychotropic agents by patients in the Department of Veterans Affairs who were given long-term antipsychotic prescriptions, by number of antipsychotics received 


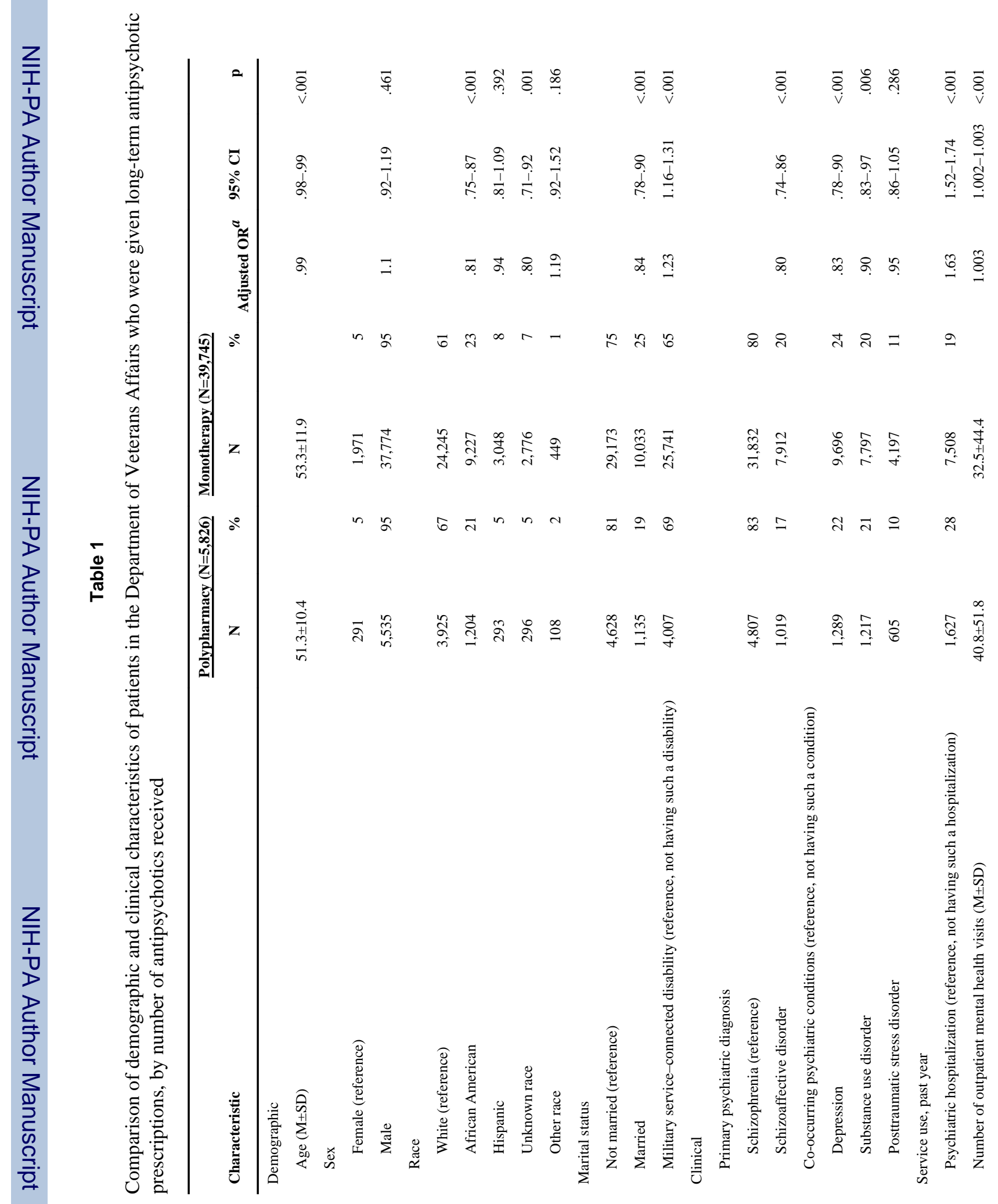




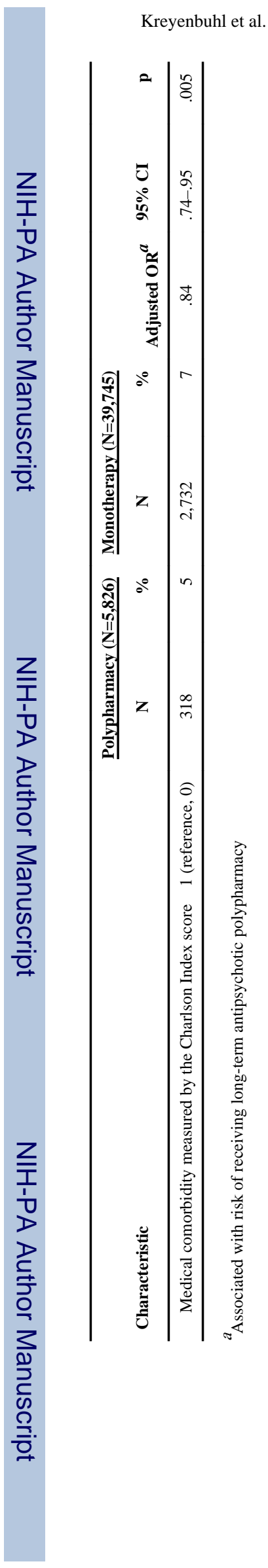

Page 12

Psychiatr Serv. Author manuscript; available in PMC 2013 June 05. 


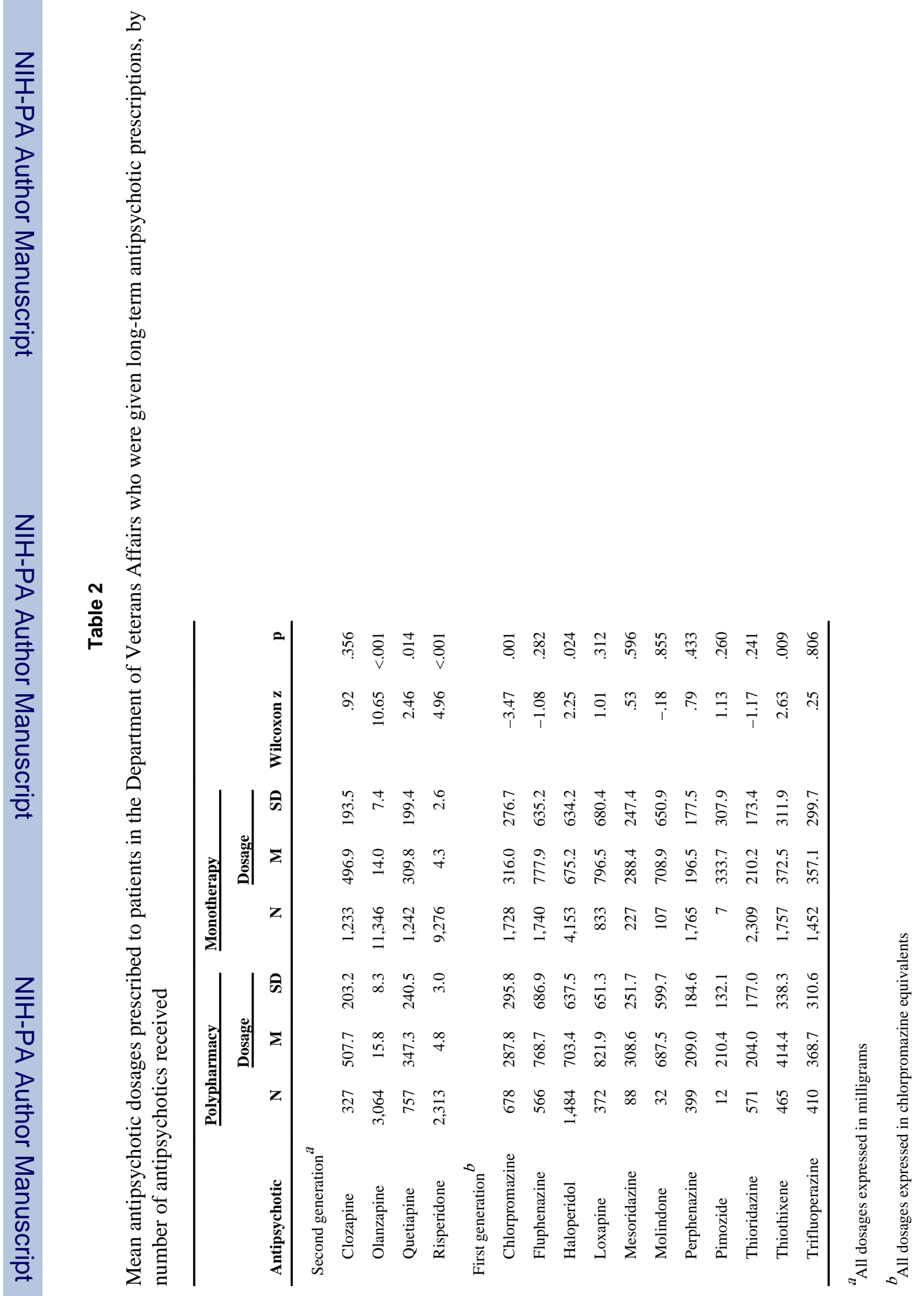

Psychiatr Serv. Author manuscript; available in PMC 2013 June 05. 